\title{
Femtosecond optical frequency combs
}

\author{
Th. Udem, R. Holzwarth, and Th. Hänsch \\ Max-Planck-Institute für Quantenoptik, Hans-Kopfermann Straße 1, 85748 Garching, Germany
}

\begin{abstract}
A laser frequency comb allows the conversion of the very rapid oscillations of visible light of some 100's of THz down to frequencies that can be handled with conventional electronics. This capability has enabled the most precise laser spectroscopy experiments yet that allowed to test quantum electrodynamics, to determine fundamental constants and to search for possible slow changes of these constants. Using an optical frequency reference in combination with a laser frequency comb has made it possible to construct all optical atomic clocks, that are now outperforming even the best cesium atomic clocks. In future direct frequency comb spectroscopy might enable high resolution laser spectroscopy in the extreme ultraviolet for the first time. Frequency combs are also used to calibrate astronomical spectrographs and might reach an accuracy that is sufficient to observe the expansion of the universe in real time.
\end{abstract}

\section{Introduction}

Frequency can be measured with by far the highest precision of all physical quantities. In the radio frequency $(\mathrm{rf})$ domain $(\omega<2 \pi \times 100 \mathrm{GHz})$, frequency counters have existed for a long time. Almost all of the most precise measurements in physics have been performed with such a counter that uses an atomic clock as a time base. To extend this accurate technique to higher frequencies, so called harmonic frequency chains have been constructed since the late 1960s $[1,2]$. In such a chain nonlinear elements produce frequency multiples (harmonics) of a given oscillator to which a subsequent oscillator is phase stabilized. Repeating this procedure several times makes it possible to multiply a reference radio frequency to much higher frequencies. Electronic phase locked loops [3] can be used to stabilize the relative phase of any kind of oscillator, even lasers, provided their intrinsic stability is sufficient so that there is no need for very rapid frequency corrections. It was not before 1996 when visible laser light was first referenced phase coherently to a cesium atomic clock using this method [4].

The disadvantage of these harmonic frequency chains was not only that these set-ups could fill several large laser laboratories at once, but that they could be used to measure a single optical frequency only. A new solution to that problem appeared at the end of the 1990s. Its central ingredient is a mode locked laser that is capable of producing very short pulses, a few femtoseconds long. Such a laser necessarily emits a very broad spectrum, comparable in width to the optical carrier frequency. The laser stores this light pulse inside its resonator for an almost unlimited duration emitting a copy of the stored pulse after each round trip time $T$ that typically lasts 1 and $10 \mathrm{~ns}$.

In the frequency domain a train of short pulses from a mode locked laser is the result of a phase coherent superposition of many continuous wave (cw) longitudinal cavity modes. These modes form a series of frequency spikes that is referred to as a frequency comb. The individual modes can be selected by phase locking other $\mathrm{cw}$ lasers to them. The modes are remarkably uniform, i.e. the separation between adjacent modes is constant across the frequency comb. This strictly regular arrangement is the most important and defining feature of a frequency comb and has first been demonstrated in [5]. The optical frequencies $\omega_{n}$ of the comb may be 
expressed as:

$$
\omega_{n}=n \omega_{r}+\omega_{C E} .
$$

Because a periodic signal in the time domain corresponds to a periodic structure in the frequency domain with inverse spacing, the modes are separated by $\omega_{r}=2 \pi / T$. They are enumerated by the mode number $n$ of some $10^{6}$ such that the frequency offset $\omega_{C E}$ lies in between 0 and $\omega_{r}$. The latter is due to the difference of the phase and group velocity inside the laser cavity and shifts the modes from being simple harmonics of the repetition rate as will be shown in the next section.

\section{The optical frequency comb}

To derive the frequency comb properties as detailed by eq. (1), it is useful to consider the electric field $E(t)$ of the emitted pulse train [6-8]. If the pulses were exact time shifted copies, $E(t)=E(t-T)$, a simple Fourier transformation would yield a strictly periodic spectrum with a mode separation of $\omega_{r}$ and a vanishing comb offset $\omega_{C E}=0$. However, this is not what is expected in a real laser. Because of intra-cavity dispersion, the group and phase velocities do not match for the pulse that is stored in the cavity. This causes the carrier wave to continuously shift with respect to the pulse envelope (see fig. 1). The copies of the pulses that exit the laser cavity through the output coupling mirror after each round trip show a pulse-to-pulse carrier envelope phase shift of $\Delta \varphi$.

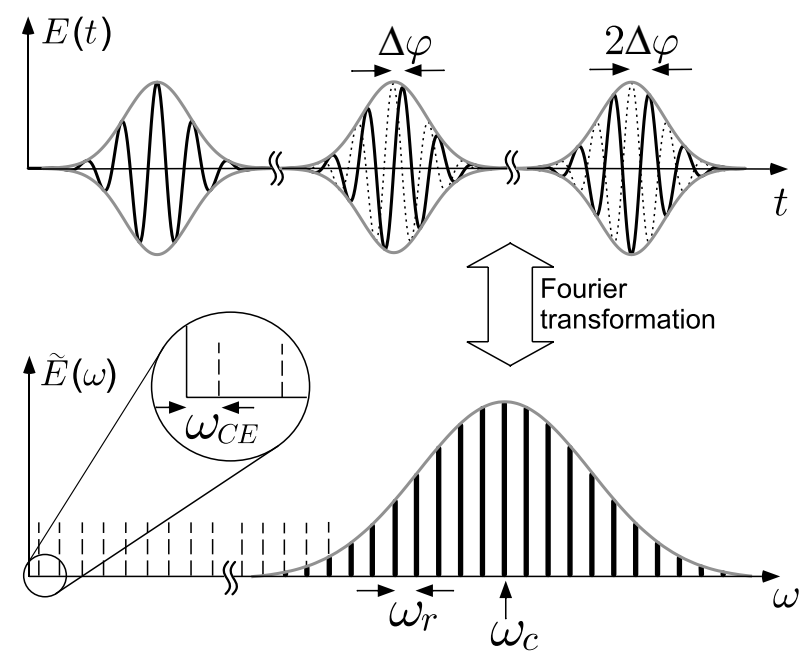

Fig. 1. Three consecutive pulses of the pulse train emitted by a mode locked laser and the corresponding spectrum. Because the optical carrier wave at $\omega_{c}$ moves with the phase velocity that is different from the group velocity that propagates the envelope, a pulse-to-pulse phase shift $\Delta \varphi$ results. In the frequency domain this corresponds to an offset frequency $\omega_{C E}=\Delta \varphi / T$ that shifts the comb as a whole from the strict harmonics of $\omega_{r}$.

The difference between phase and group velocity is determined by the dispersion properties of the cavity as a whole. The remarkable property of Kerr-lens mode locked lasers is that this difference is the only effect of intra-cavity dispersion on the pulse train. Higher order dispersive terms that would continuously deform the pulse are strongly suppressed so that the pulse can circulate undistorted for virtually unlimited time. This long term coherence is the intrinsic reason for the very precise uniformity of the mode spacing expressed by eq. (1). In fact, as of today no deviation from this expression has been detected $[15,20]$.

To analyze this situation the pulse train is most conveniently written as an optical carrier wave propagating with its phase velocity at $\omega_{c}$ modulated by the pulse envelope function $A(t)$ 
that propagates with its group velocity. The observed strict periodicity of the latter is expressed by demanding $A(t)=A(t-T)$ with the pulse repetition time $T=2 \pi / \omega_{r}$. To obtain the spectrum of the pulse train the envelope can be re-expressed through a Fourier series

$$
E(t)=A(t) e^{-i \omega_{c} t}+\text { c.c. }=\sum_{q} A_{q} e^{-i\left(\omega_{c}+q \omega_{r}\right) t}+\text { c.c. }
$$

where $A_{q}$ are Fourier components of $A(t)$. This equation represents a comb of laser frequencies with spacing $\omega_{r}$ that is shifted as a whole from the harmonics of $\omega_{r}$, just as in eq. (1). The only difference being the numbering of modes which clearly is a matter of convention.

In a real laser the pulses may be chirped. That is their carrier frequency varies across the pulse. Such a chirp from a laser is periodic with the repetition rate otherwise the carrier wave would increase/decrease indefinitely. For this reason the chirp can be accounted for by allowing a complex envelope function $A(t)$ leaving all of the above arguments valid.

Another obvious difference of the above model to a real laser is that neither the carrier wave nor the pulse train will be free of noise. To take this into account the Fourier series in eq. (2) has to be replaced by a convolution of the carrier wave spectrum with the spectrum of the pulse envelope [21]. Provided the carrier wave possesses a suitably narrow line width and the noise in the repetition rate is low enough in order to maintain the comb structure, individual modes can be separated. This condition is easily satisfied, even with a free running mode locked laser. The noise of the frequency comb will be taken out by phase locked loops as detailed below, in order to stabilize both radio frequencies in eq. (1) relative to a precisely known radio frequency reference. Usually only long slow fluctuations $(\sim 100 \mathrm{~Hz})$ have to be compensated for the repetition rate while slightly more bandwidth is required for the carrierenvelope frequency. In the phase locked condition the above derivation remains valid when averaging over time scales much longer than the inverse bandwidths of the phase locked loops.

It is easy to show that a grating with sufficient resolution to resolve the frequency comb would be at least as large as the laser cavity, which appears unrealistic for most mode locked lasers. Fortunately for frequency metrology applications it is not necessary to resolve individual modes in the optical domain.

The spectral width of femtosecond light pulses produced by a mode locked laser can be significantly broadened by virtue of self phase modulation in optical fibers. This process, whose origin is an intensity dependent refractive index, is described in the time domain by the optical Kerr effect (the same effect used to operate a Kerr lense mode locked laser). After propagating a length $l$ the intensity dependent refractive index $n(t)=n_{o}+n_{2} I(t)$ leads to a self induced phase shift of

$$
\Phi(t)=-n_{2} I(t) \omega_{c} l / c \quad \text { with } \quad I(t)=|A(t)|^{2} .
$$

This time dependent phase shift leads to a frequency modulation that is proportional to the time derivative of the self induced phase shift $\dot{\Phi}(t)$. Self-phase modulation modifies the envelope function according to

$$
A(t) \longrightarrow A(t) e^{i \Phi(t)}
$$

Because $\Phi(t)$ has the same periodicity as $A(t)$ the comb structure of the spectrum, as previously derived, is not affected.

In an optical fiber this process is more efficient than in bulk material, even though the nonlinear coefficient in fused silica is comparatively small. This is because the fiber core carries a high intensity over an extended length. However, dispersion causes the pulse to broaden temporarily so that the large peak intensity required for that process can come to a halt after a few $\mathrm{cm}$ of propagation length. Optical fibers that partially compensate that effect can be much more effective in terms of spectral broadening. In the near infrared one can employ the zero of group velocity dispersion of silica around $1.3 \mu \mathrm{m}$ that can be shifted to other wavelength by a properly designed refractive index profile of the wave guide. In addition doping can be used to increase the non-linearity. This approach is generally used with fiber based mode locked lasers that operate in this spectral region. For wavelengths around $800 \mathrm{~nm}$ where titanium sapphire mode locked lasers operate photonic crystal fibers (or micro structured fibers) may be used. Such a fiber uses an array of air holes that surround the fiber core and run the length of fiber 
to change its effective dispersion [9]. Some lasers can reach an octave spanning spectrum even without using external self-phase modulation. This became possible with enhanced intra-cavity self-phase modulation [10] and/or by using extremely broad band intra-cavity mirrors [11]. These titanium sapphire lasers are an advantage because they avoid problems associated with mechanical stability when coupling into the tiny core of a photonic crystal fiber.

\section{Self-referencing}

To stabilize the frequency comb it is first of all necessary to generate the two radio frequencies $\omega_{r}$ and $\omega_{C E}$ that make up the frequency comb in order to stabilize them relative to some radio frequency reference. Whereas the pulse repetition frequency may be detected anywhere in the laser beam, the generation of the carrier envelope frequency is more involved. In fact generating $\omega_{C E}$ became possible only in 1999 by beating different harmonics (3.5th and 4th) of different components of the comb [12]. As this first demonstration involved considerable effort to generate the required harmonics, the task simplified dramatically with the availability of octave spanning frequency combs [13-15].
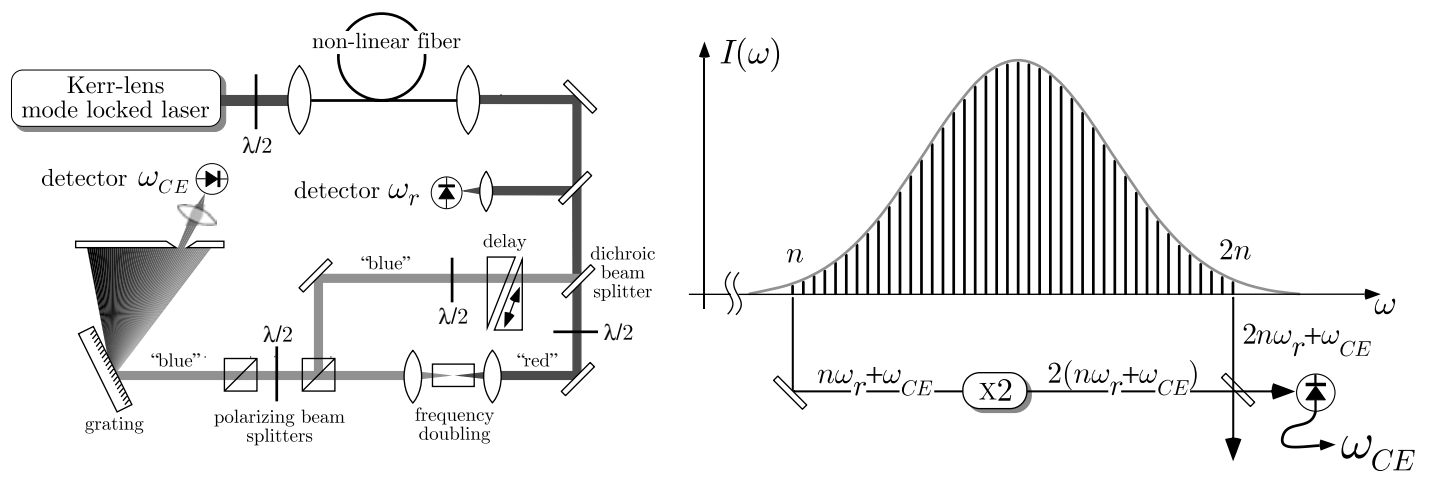

Fig. 2. The carrier envelope offset frequency $\omega_{C E}$ that displaces the modes of an octave spanning frequency comb from being exact harmonics of the repetition rate $\omega_{r}$ is measured by frequency doubling some modes at the "red" side of the comb and beat them with modes at the "blue" side. The right side figure shows this scheme in the frequency domain while the left part sketches the beam paths in the actual set-up.

The so called $f$-to- $2 f$ self-referencing uses a group of modes from the low frequency end of the comb around $n_{L} \omega_{r}+\omega_{C E}$ that is frequency doubled in a nonlinear crystal and super imposed with another group of modes around $n_{H}$ at the high frequency end of the comb. This generates signals at the difference frequency of two optical waves on a photo detector: $2\left(n_{L} \omega_{r}+\omega_{C E}\right)-n_{H} \omega_{r}+\omega_{C E}=\left(2 n_{L}-n_{H}\right) \omega_{r}+\omega_{C E}$. These beat frequencies are created for all possible combinations of $n_{L}$ and $n_{H}$ that lie within the bandwidth of the photo detector. One can restrict the number of signals to precisely one by using a rf low pass filter. If this filter has a cut off frequency of $\omega_{r} / 2$ only the beat note at $\omega_{C E}$, belonging to all combinations with $2 n_{L}-n_{H}=0$, remains. The octave spanning comb ensures that these combinations exist, i.e. that the modes $n_{L}$ and $n_{H}$ with $n_{H}=2 n_{L}$ are active modes of the comb. Knowing both, $\omega_{C E}$ and $\omega_{r}$, means that frequencies of all modes according to eq. (1) are known and may be used for optical frequency measurements. This is accomplished by creating another beat note $\omega_{b}$ between an unknown laser at $\omega_{L}$ and a nearby mode of the comb. The frequency of that laser can then be determined by $\omega_{l}=n \omega_{r}+\omega_{C E}+\omega_{b}$ (where $\omega_{C E}$ and $\omega_{b}$ may be negative). The mode number $n$ may be determined by a coarse measurement of $\omega_{l}$, say with a wavelength meter or by repeating the measurement with slight variations in $\omega_{r}$ [16]. A typical set-up for $f$-to- $2 f$ self-referencing sketched in fig. 2 . 
By adjusting the resonator length, through a piezo-electric transducer, the repetition rate can be adjusted. For a typical laser the intrinsic stability of the repetition rate is good enough such that the attainable bandwidth of the transducer is by far sufficient to keep it in phase with a reasonable rf reference. The other comb parameter is determined by both, the phase and group velocity, of the stored pulse. It was shown that for a soliton like laser such as the titanium sapphire Kerr-lense mode locked laser, the group- and phase velocity depend in a different way on the peak intensity of the stored pulse [17]. Therefore one can adjust the pulse to pulse slippage of the carrier envelope phase, and thereby $\omega_{C E}$ by controlling the average laser power. For titanium sapphire and fiber mode locked laser this can be done by controlling the power of the pump laser. The two controls are not completely independent. However, any modification of the frequency that maintains the comb properties can be characterized by a fix-point close to the unaffected mode [18]. It turns out that the fix-point with respect to pump laser modulation is usually located close to the center of the frequency comb $[18,19]$. For this reason using the pump power and the cavity length always changes both degrees of freedom of the frequency comb. Keeping the servo bandwidths for $\omega_{r}$ as low as possible and for $\omega_{C E}$ as high as possible usually de-couples the two servo systems. Having the two phase servo systems operational ensures, by virtue of eq. (1) and the large integer $n$, that the radio frequencies $\omega_{r}$ and $\omega_{C E}$ are phase coherently up-converted to the optical region.

To investigate the integrity of the frequency comb and to check for possible systematic deviations several tests have been performed. Two independent frequency combs have been found to agree at a level of $5.1 \times 10^{-16}[15]$ and $1.3 \times 10^{-17}$ [20]. Even though Doppler shifts due to mirror motions caused by thermal expansion set in at this level, no systematic shifts have been found yet.

\section{Applications}

A frequency comb can be used to phase coherently connect the radio frequency domain with the optical domain. This is enabled by the large integer $n\left(\approx 10^{5} \ldots 10^{6}\right)$ that the pulse repetition frequency is multiplied with. As sketched in fig. 3 three interesting frequency conversions are possible with the frequency comb. Locking the radio frequencies $\omega_{r}$ and $\omega_{C E}$ to a Cs clock references the optical modes to the SI second in order to determine optical transition frequencies of interest with the utmost precision. Using the repetition rate as a reference to stabilize the carrier envelope frequency allows to divide an optical frequency down to the radio frequency domain or to directly measure optical frequency ratios [22].
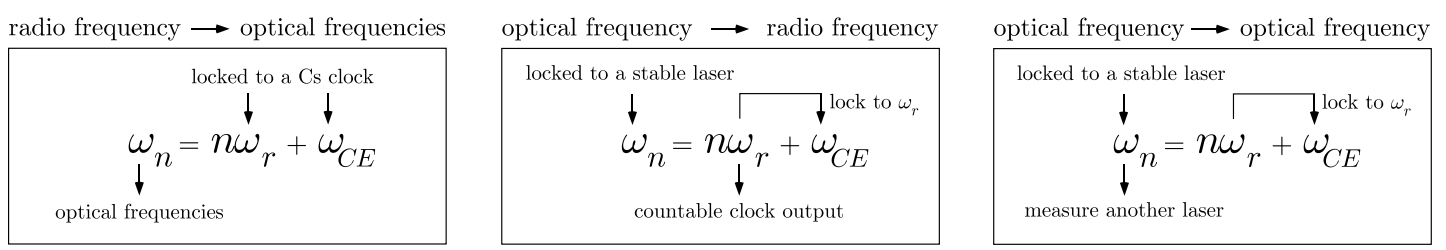

Fig. 3. Frequency conversions possible with the frequency comb. Left: by locking to radio frequencies $\omega_{r}$ and $\omega_{C E}$ of the comb to a Cs atomic clock the optical modes $\omega_{n}$ may be used to determine atomic transition frequencies in units of Hz. Center: in reverse operation the comb is referenced to an optical transition extracting a pre-scaled radio frequency copy. Right: phase coherent optical-optical frequency ratios are also measured.

\subsection{Optical clocks}

An optical clock consists, like most other clocks, of an oscillator that defines the ticks in time and a counter that keeps track of these cycles. In a cesium clock for example the ground state hyperfine splitting of the ${ }^{133} \mathrm{Cs}$ atom is used. An electronic counter advances the second hand 
every time the counter has received 9192631770 oscillations. This number was chosen when the SI second was redefined for the last time in 1967 to match with the previous definition. If we look at the history of time keeping and compare clocks as different as sun dials, pendulum clocks and quartz clocks it is obvious that they get more accurate as the oscillation frequency increases. This is simply because a higher oscillation frequency slices time into smaller pieces. Since the end of the 1950s the cesium $9.192 \ldots$ GHz oscillator is used. With the frequency comb at hand one has a simple tool to count up 5 orders of magnitude faster. In fact one can show that any clock limited by quantum noise will possess an instability that decreases with the inverse of the averaging time and is proportional to the observed line width divided by the transition frequency [25]. For practical reasons one cannot simply pick a transition with an arbitrary small line width, because this will at the same time increase the required observation time. For this reason optical oscillators have been envisioned to be used in clocks for a long time. For a well chosen transition the systematic uncertainties are expected to be as low as $10^{-18}$.

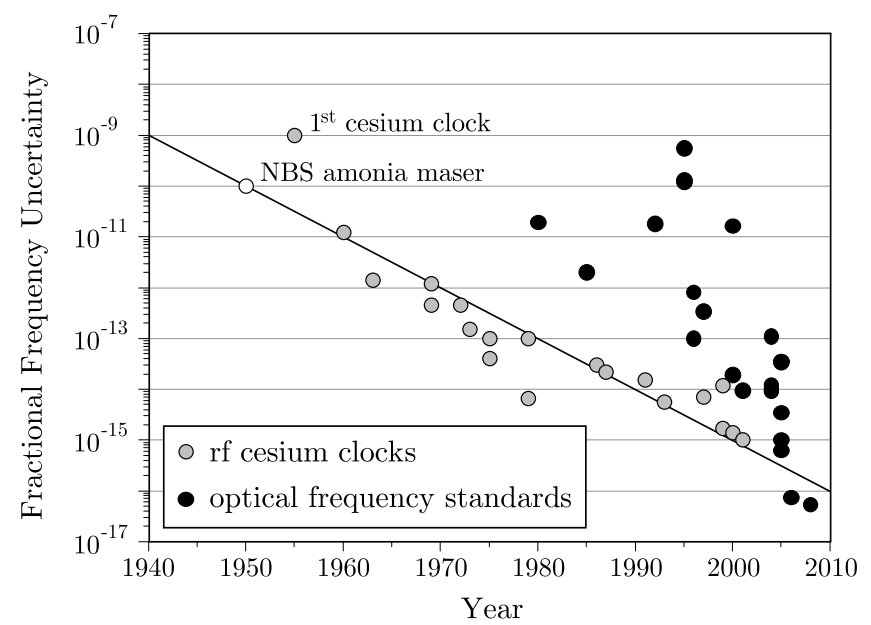

Fig. 4. Evolution of radio frequency (rf) and optical frequency standards. All optical measurements since 2000 have been conducted with frequency combs, giving a significant boost to the field. Now the best optical clocks are outperforming even the best cesium atomic clocks [26].

After tremendous advances in laser spectroscopy in the 1980s and 1990s that ultimately resulted in trapped atom and ion standards (see for example [23,24]), a reliable optical clock work mechanism was the missing piece for completion of the all optical atomic clock. The best of these new clocks are now surpassing the accuracy of even the best Cs fountain clocks. To demonstrate such a performance a comparison with another optical clock is required. Recently T. Rosenband and co-workers at NIST have succeeded with such a measurement that shows the stability of two optical clocks based on single trapped $\mathrm{Hg}$ and $\mathrm{Al}$ ions. The frequency ratio measured as sketched at the right hand side of fig. 3 has been found to be reproducible within $5.2 \times 10^{-17}[26]$.

An overview of the evolution of radio frequency (cesium) clocks versus optical standards over the last decades is presented in fig. 4 . Whereas the cesium atomic clocks have steadily improved by an order of magnitude per decade, the optical frequency standards have significantly gained in pace since the introduction of frequency combs. The existing optical clocks are still quite complicated devices and various atoms and ions are investigated. One expects this process to continue for some time before a particular transition is chosen to re-define the SI second.

\subsection{Basic research}

The possibility to readily count optical frequencies has opened up new experimental possibilities. High precision measurements on hydrogen have allowed for improved tests of the predictions 
of Quantum Electrodynamics (QED) and the determination of the Rydberg constant [27]. As the simplest of all stable atomic systems the hydrogen atom provides the unique possibility to confront theoretical predictions with experimental results. The narrowest line starting from the $1 \mathrm{~S}$ ground state in hydrogen is the $1 \mathrm{~S}-2 \mathrm{~S}$ two-photon transition with a natural line width of $1.3 \mathrm{~Hz}$ at transition frequency of $2466 \mathrm{THz}$. To explore the full capacity of this transition, a frequency measurement is imperative. In fact this task motivated the construction of the first self-referenced frequency comb $[12,14]$. At that time the frequency comb was referenced to a transportable cesium fountain clock from BNM-LPTF (now LNE-SYRTE), Paris [28]. Through the introduction of the frequency comb the uncertainty of this measurement became limited by the spectrometer to about 2 parts in $10^{14}$. Lacking a suitable laser cooling method is a particular problem for the light hydrogen atom so that the limits are currently set by how well the velocity and angular distribution of the $6 \mathrm{~K}$ atomic beam is understood.

A new measurement in 2003 did not yield an improvement but was useful to set an upper limit on a possible small variation of the fine structure constant as well as the strong interaction constant [29]. The question of slowly drifting fundamental constants was first raised in 1937 by P.A.M. Dirac, where he speculated that such a drift would be synchronized with the expansion of the universe [30]. The traditional way to search for such a phenomenon is to determine the value of say the fine structure constant as it was effective billions of years ago by astronomical or geological observations. With the frequency combs it is now possible to compete in sensitivity with these observation with laboratory measurements even though the look back time is typically about 9 orders of magnitude smaller.

In the first round of these experiments the hydrogen $1 \mathrm{~S}-2 \mathrm{~S}$ [29] and transitions in single trapped $\mathrm{Hg}$ [31] and $\mathrm{Yb}$ ions [32] were used by comparing them with the best cesium atomic clocks. Since the ${ }^{133} \mathrm{Cs}$ ground state hyperfine splitting is determined by the nuclear structure, the strong interaction is mainly scaling the reference frequency. It is therefore impossible to distinguish a variation of the fine structure constant from the strong coupling constant using a single atomic system. If such a variation actually takes place, grant unified theories predict a synchronization of the various coupling constants [54] such that it may cancel out from the observation of a single system. Fortunately the $\mathrm{H}, \mathrm{Yb}^{+}$and $\mathrm{Hg}^{+}$possess different dependences on the fine structure constant so that the contributions of the coupling constants can be separated [33].

An even better way to detect a drifting fine structure constant is to observe optical frequency ratios as discussed in section 3. The above mentioned comparison of the $\mathrm{Hg}^{+}$and $\mathrm{Al}^{+}$optical clocks provides such a unique possibility. After only one year of observation this frequency ratio puts the smallest limit of $5.2 \times 10^{-17} \mathrm{yr}^{-1}$ on the drift of the fine structure constant yet [26] Remarkably, the precision of these laboratory measurement makes it possible to reach the same sensitivity within a few years of monitoring that astronomical and geological observations require billions of years of look back time. In the laboratory systematics can be investigated or challenged, and if in doubt, experiments may be repeated given the relative short time intervals. So far none of the laboratory tests have been able to detect a temporal variation of the fine structure constant. Geological and astronomical observations may be affected by systematic uncertainties that lead to partially contradicting results (see ref. [34-36] for results on quasar absorption and $[37,38]$ geological data). It should however be pointed out that laboratory tests operate on a different epoch than astronomical and geological observations so that the data may not be directly comparable.

A very important application for improved atomic clocks in basic research could be to test or refine general relativity which still poses one of the major problems in physics as it refuses proper quantization. It is possible that small, yet undetected, deviations from the classical theory of gravity give valuable hints on how to proceed with quantization.

\subsection{High harmonics and $\mathrm{He}^{+}$}

Laser like pulsed emission of very short wavelength can be produced with a method generally referred to as high harmonic generation (HHG) [40]. For this purpose one focuses infrared femtosecond pulses in a gas target employing its extreme nonlinear response when subject to 
intensities exceeding $\sim 5 \times 10^{12} \mathrm{~W} / \mathrm{cm}^{2}$. In most HHG experiments a nozzle emitting a jet of noble gas atoms is placed near the focus of an amplified femtosecond pulses train to produce up to the $\sim 1000$-th harmonic [41] in a nearly diffraction limited laser like beam that travels co-linearly with the generating pulse train.

We expect that each of these harmonics is made up of a frequency comb so that direct frequency comb spectroscopy [42] should be possible in the extreme ultra violet. It can be shown that for a two-photon transition driven with the modes of a frequency comb, the modes will add up pair wise to the same transition energy. In this case the effective laser line width corresponds to the line width of a single mode while the transition probability is calculated from the power of all modes [44]. There are several conditions for the HHG radiation to be useful for this type of high resolution spectroscopy: (i) the HHG process must be coherent in order to support the mode structure of a frequency comb. (ii) the mode spacing (repetition rate) must be sufficiently large so that the ion or atom can separate out individual modes. (iii) the power of the HHG radiation has to be sufficient to obtain a detectable transition rate.

Even though there is lots of evidence that the process of HHG is fully coherent [43], no direct detection of the existence of a frequency comb has been performed yet beyond the third harmonic. The second condition states that the pulse repetition rate must be much larger than the observed line width. This requirement is contradicting the requirement for high peak intensities necessary to drive HHG. A large pulse repetition rate distributes the limited average power on many pulses per second. A promising route to solve this issue is to generate HHG's inside an enhancement resonator. Harmonics with up to $110 \mathrm{MHz}$ repetition rate where generated this way $[45,46]$. In the first generation of these set-ups the extracted extreme ultraviolet (XUV) power was by far not sufficient to be useful for high resolution laser spectroscopy.

Thanks to the highly non-linear character of the process, moderate improvements already led to orders of magnitude more power which is now getting into a regime with a useful excitation rate say at the $1 \mathrm{~S}-2 \mathrm{~S}$ transition in $\mathrm{He}^{+}[47,48]$. This very sharp transition takes place with two $60 \mathrm{~nm}$ photons. Since $\mathrm{He}^{+}$is iso-electronic with the hydrogen atom, its energy levels can be calculated with the same accuracy using QED. Besides demonstrating an all new spectroscopy tool, $\mathrm{He}^{+}$can solve several experimental and theoretical problems currently encountered with hydrogen. Because of the very unfavorable laser wavelengths, hydrogen has never been laser cooled. Due to its small mass even at a temperature of a few Kelvin reachable with a cryostat, it is difficult to keep the hydrogen atoms inside the laser beam for more than one millisecond. This causes a set of systematic problems that has clamped the accuracy of the $1 \mathrm{~S}-2 \mathrm{~S}$ transition to about 1 part in $10^{14}$ for several years. Since $\mathrm{He}^{+}$is charged it may be held in a trap extending to virtually unlimited interaction time. Trapped He ions can further be sympathetically cooled by co-storing other ions such as $\mathrm{Mg}^{+}$for which cooling lasers are readily available.

On the theoretical side contributions to the energy levels with higher powers of the nuclear charge $Z$ will generally allow a more sensitive test of QED. The Lamb shift corrections scale in lowest order as $Z^{4}$ with the $Z^{6}$ terms currently under consideration. For this reason the same interesting physics is investigated at significantly lower resolution by going from hydrogen to $\mathrm{He}^{+}$.

\subsection{Carrier envelope phase}

The application of the frequency combs has also enabled advances in another field as it allows the possibility for stabilizing the carrier envelope phase [7,49]. According to fig. 1 a pulse train with a vanishing $\omega_{C E}$ has a fixed phase of the carrier with respect to the envelope. This means that all the pulses have the same electric field. With the technique of self referencing this can be readily accomplished. However, even though the electric field transients of the pulses are identical in this case, it is unknown what value the carrier envelope phase actually assumes upon stopping its pulse to pulse slippage.

To detect the value of the carrier envelope phase, very short pulses that drive processes which depend on the electric field in high order are used. Figure 5 shows two extreme values of the carrier envelope phase that correspond to a "cos" and a "sine" pulse. Close observation of the field transients reveals that the peak electric field slightly depends on the carrier envelope 
phase. In addition the duration of the optical carrier cycle changes slightly due to the steep pulse envelope when measured say between two field maxima. These effects are largely enhanced if the pulses are short and can be detected using HHG's or above threshold ionization [51].

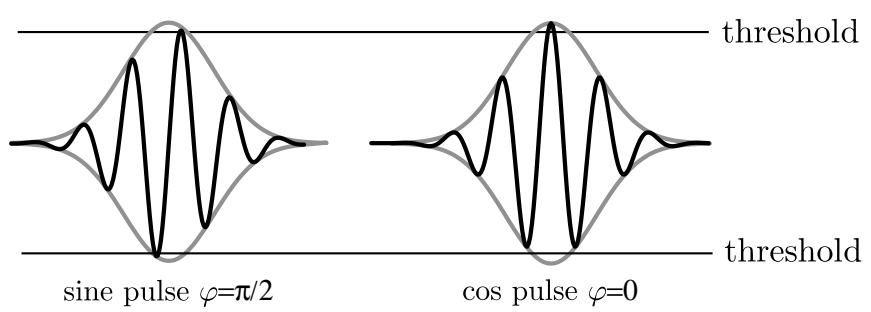

Fig. 5. Very short infrared pulses driving a very non-linear process such as high harmonic generation close to the short wavelength cut-off. If the intensity and/or the non-linearity is well chosen such that it sets in at a certain threshold, a "sine" pulse will produce a spectrum that is periodic with the infrared carrier frequency because the threshold is reached twice per pulse (left). In contrast the "cos" pulse (right) produces a single isolated harmonic pulse that is much shorter than an optical cycle of the infrared driving pulse [53].

Two important aspects are realized from this: for "cos" driving pulse the high harmonic pulse is much shorter than one cycle of the generating field. So by stabilizing the carrier envelope phase to the proper value single isolated attosecond could be produced for the first time [50]. Secondly the high harmonic spectrum reveals whether the carrier envelope phase has been fixed at the proper value or not. A "sine" drive pulse creates two high harmonic pulses per laser pulse, that have almost but not quite the time separation given by the carrier frequency. For this reason the spectrum shows well separated harmonic peaks even at the shortest wavelength that are generated. In contrast to that, a "cos" produces a shifted harmonic spectrum that looses its periodicity in this region. This is because it belongs to a single isolated attosecond pulse [53].

Not only that the detection and stabilization of the carrier envelope phase allowed the production of attosecond pulses for the first time, it also allowed to completely recover the electric field transients of ultra short pulses. The work in that direction relied on measuring the pulses autocorrelation which, together with the determination of the carrier envelope phase via spectral analysis of high harmonics allows the calculation of the field transient [50]. A more direct measurement uses cross correlation between attosecond pulses generated the way described above with the driving pulses. In this way the field transient of the latter can be sampled with a temporal resolution significantly shorter than one optical cycle [52].

\subsection{Optical frequencies in astronomy}

In connection with the cosmological search for a variation of fundamental constants, optical frequency measurements are useful on samples in the sky and on Earth to compare with. To detect extrasolar planets the most powerful method has been to measure the changing recoil velocity of its star during the orbital period. These recoil velocities are rather small unless a massive planet in close orbit is considered. This is the reason why mostly "hot Jupiters" are among the roughly 200 extrasolar planets detected so far. The lightest of those planets possesses about 10 Earth masses. The wobble that our planet imposes on the motion of our Sun has a velocity amplitude of only $v_{E}=9 \mathrm{~cm} / \mathrm{sec}$ with a period of one year of course. Because Earth and Sun maintain their distance as they go around their common center of mass, this motion is invisible from Earth. On the other hand it can be detected at other stars, where it is superimposed with the center of mass motion of that system and the motion of the Earth around the Sun. To detect Earth-like planets that orbit Sun-like stars with the recoil velocity method, a relative Doppler shift of $v_{E} / c=3 \times 10^{-10}$ needs to be measurable. Converted to visible 
radiation of say $500 \mathrm{THz}$ this requires a resolution of $150 \mathrm{kHz}$ and the same reproducibility after half the orbital time.

Spectral lines from atoms and ions from interstellar clouds and the surface of stars are subject to strong line broadening due to collisions and the Doppler broadening of typically several $\mathrm{GHz}$ due to their thermal motion. They are measured with telescopes like the Very Large Telescope operated by the European Southern Observatory which can be connected to an Echelle type spectrometer with high resolution. Given these rather broad lines, the required spectral or velocity resolution can be obtained only by using the statistics of many lines observed simultaneously. For calibration the rather irregular line spectrum of Th-Ar lamps have been used. Using a frequency comb for this purpose appears to be the optimum tool, both in terms of providing an equidistant dense calibration and for allowing long term reproducibility that goes well beyond the typical life time of an individual spectrometer [55]. The latter property derives from the possibility to reference to a precise clock. In this case even a simple GPS disciplined rubidium clock suffices for the required $3 \times 10^{-10}$ reproducibility to detect Earth-like extrasolar planets.

Yet another important application of precise astronomical spectroscopy is to probe the cosmic dynamics as a whole. So far the expansion dynamics is only derived from snap shots of red shift distributions assuming the validity of general relativity for its interpretation. Frequency comb calibrated spectrometers could become accurate enough such that the presumed acceleration rate of the Universe can be observed in real time.

\section{References}

1. L.O. Hocker, A. Javan, D.R. Rao, L. Frenkel, Appl. Phys. Lett. 10, 147 (1967)

2. K.M. Evenson, J.S. Wells, F.R. Petersen, B.L. Danielson, G.W. Day, Appl. Phys. Lett. 22, 192 (1973)

3. See for example: F.M. Gardener, Phaselock Techniques (John Wiley \& Sons, New York, 1979)

4. H. Schnatz, B. Lipphardt, J. Helmcke, F. Riehle, G. Zinner, Phys. Rev. Lett. 76, 18 (1996)

5. Th. Udem, J. Reichert, R. Holzwarth, T.W. Hänsch, Opt. Lett. 24, 881 (1999)

6. Th. Udem, Thesis, Ludwig-Maximilians Universität, Munich, Germany (1997)

7. J. Reichert, R. Holzwarth, Th. Udem, T.W. Hänsch, Opt. Commun. 172, 59 (1999)

8. Th. Udem, R. Holzwarth, T.W. Hänsch, Nature 416, 233 (2002)

9. P.St.J. Russell, Science 299, 358 (2003)

10. R. Ell, et al., Opt. Lett. 26, 373 (2001)

11. L. Matos, et al., Opt. Lett. 29, 1683 (2004)

12. Th. Udem, J. Reichert, R. Holzwarth, T.W. Hänsch, Proceedings of the 1999 Joint Meeting of the European Frequency and Time Forum (EFTF99) and the IEEE International Frequency Control Symposium (FCS99), Vol. 2 (1999) pp. 620-625

13. S.A. Diddams, et al., Phys. Rev. Lett. 84, 5102 (2000)

14. M. Niering, et al., Phys. Rev. Lett. 84, 5496 (2000)

15. R. Holzwarth, et al., Phys. Rev. Lett. 85, 2264 (2000)

16. R. Holzwarth, et al., Appl. Phys. B 73, 269 (2001)

17. H.A. Haus, E.P. Ippen, Opt. Lett. 26, 1654 (2001)

18. N. Haverkamp, H. Hundertmark, C. Fallnich, H.R. Telle, Appl. Phys, B 78, 321 (2004)

19. D.R. Walker, Th. Udem, Ch. Gohle, B. Stein, T.W. Hänsch, Appl. Phys. B 89, 535 (2007)

20. S.A. Diddams, L. Hollberg, L.S. Ma, L. Robertsson, Opt. Lett. 27, 58 (2002)

21. Th. Udem, F. Riehle, Rev. Nuovo Cimento 30, 563 (2007)

22. S.A. Diddams, et al., Science 293, 825 (2001)

23. P. Gill, H. Margolis, Phys. World, 35 (2005)

24. S.A. Diddams, J.C. Bergquist, S.R. Jefferts, C.W. Oates, Science 306, 1318 (2004)

25. L. Hollberg, et al., IEEE J. Quant. Electr. 37, 1502 (2001)

26. T. Rosenband, et al., Science 319, 1808 (2008)

27. B. de Beauvoir, et al., Eur. Phys. Lett. D 12, 61 (2000)

28. G. Santarelli, et al., Phys. Rev. Lett. 82, 4619 (1999)

29. M. Fischer, et al., Phys. Rev. Lett. 92, 230802 (2004)

30. P.A.M. Dirac, Nature (London) 139, 323 (1937) 
31. S. Bize, et al., Phys. Rev. Lett. 90, 150802 (2003)

32. E. Peik, et al., Phys. Rev. Lett. 93, 170801 (2004)

33. M. Zimmermann, et al., Laser Phys. 15, 997 (2005)

34. J.K. Webb, et al., Phys. Rev. Lett. 87, 091301 (2001)

35. R. Srianand, et al., Phys. Rev. Lett. 92, 121302 (2004)

36. M.T. Murphy, et al., [arXiv:astro-ph/0612407v1] and [arXiv:astro-ph/0611080v3]

37. Y. Fujii, et al., Nucl. Phys. B 573, 377 (2000)

38. S.K. Lamoreaux, J.R. Torgerson, Phys. Rev. D 69, 121701(R) (2004)

39. T. Fortier, et al., Phys. Rev. Lett. 98, 070801 (2007)

40. For a review of the subject see for example: J.G. Eden, Prog. Quant. Electron. 28, 197 (2004)

41. J. Seres, Nature 433, 596 (2005)

42. P. Fendel, S.D. Bergeson, Th. Udem, T.W. Hänsch, Opt. Lett. 32, 791 (2007)

43. M. Bellini, T.W. Hänsch, Appl. Phys. B 65, 677 (1997)

44. Ye.F. Baklanov, V.P. Chebotayev, Appl. Phys. Lett. 12, 97 (1977)

45. C. Gohle, et al., Nature 436, 234 (2005)

46. R.J. Jones, K.D. Moll, M.J. Thorpe, J. Ye, Phys. Rev. Lett. 94, 193201 (2005)

47. A. Ozawa, et al., Phys. Rev. Lett. (in press)

48. D.C. Yost, T.R. Schibli, J. Ye, Opt. Lett. 33, 1099 (2008)

49. D.J. Jones, et al., Science 288, 635 (2000)

50. A. Baltuška, et al., Nature 421, 611 (2003)

51. G.G. Paulus, et al., Phys. Rev. Lett. 85, 253004 (2000)

52. E. Goulielmakis, et al., Science 305, 1267 (2004)

53. R. Kienberger, et al., Nature 427, 817 (2004)

54. X. Calmet, H. Fritzsch, Phys. Lett. B 540, 173 (2002)

55. M.T. Murphy, et al., Mon. Not. R. Astron. Soc. 380, 839 (2007) 